\title{
CLUSTER SETS OF NETS
}

\section{B. J. PETTIS}

We give here a few simple results on cluster sets of nets in topological spaces with some applications to real variables and elementary topological algebra.

If $\mu$ is a net in topological $X$ and $\lim \sup \mu$ denotes the set of its cluster points, then $f(\lim \sup \mu) \subset \lim \sup f(\mu)$ for any continuous $f$ on $X$; the inclusion may be proper. Theorem A asserts that the two sets are equal if every subnet of $\mu$ clusters and if the range of $f$ is Hausdorff. This fact, curiously enough, gives deeper insight into certain elementary situations in real variables and is a useful device in topological algebra, as shown in the discussion after Corollary 2 . Theorem $\mathrm{D}$ concerns nets in uniform spaces and may be considered to be the elementary principle behind Duhamel theorems. This theorem and Corollaries 1 and 2 (known to the author since 1953) were referred to in an abstract [4].

A net in $X$ is any function having a directed set for its domain and for its values nonvoid subsets of the set $X$. A net $\mu$ is finally in $G$ if $\{d: \mu(d) \subset G\}$ is a final segment of the domain of $\mu$ ("final segment" here meaning a subset containing all the successors of some element), and repeatedly intersects $G$ if $\{d: \mu(d) \cap G \neq \varnothing\}$ is cofinal. Given two nets $\mu$ and $\nu$ in $X$, we call $\nu$ a subnet of $\mu$ if for each final segment $I$ of the domain of $\mu$ there is a final segment $J$ of the domain of $\nu$ such that for each $e$ in $J$ there is some $d$ in $I$ such that $\nu(e) \subset \mu(d)$. A basic fact is that any net has a universal point subnet, i.e., a subnet every value of which is a singleton set and which, for each set $G$ in $X$, is either finally in $G$ or finally in the complement $X \backslash G$.

When $X$ is topological we define $\mathcal{F}(\mu)$ to be $\{F: F \subset X, F$ closed, $\mu$ is finally in $F\}$, and $\lim \sup \mu$ to be $\bigcap\{F: F \in \mathcal{F}(\mu)\}$. Equivalently, $x$ is in $\lim \sup \mu$ iff every neighborhood of $x$ is repeatedly intersected by $\mu$ and iff $x$ is an adherent point of $\mathcal{F}(\mu)$. If $\mu$ finally lies in each neighborhood of $x, \mu$ converges to $x$. Some facts: (i) if $\nu$ is a subnet of $\mu$ then $\mathcal{F}(\mu) \subset \mathcal{F}(\nu)$ and $\lim \sup \nu \subset \lim \sup \mu$; (ii) if $\mu$ is finally compact, meaning that $\mathcal{F}(\mu)$ has a compact element, then $\lim \sup \mu \neq \varnothing$; (iii) if $f$ on $X$ is continuous then $f(\lim \sup \mu) \subset \lim \sup f(\mu)$ for every net $\mu$ in $X$; (iv) $x \in \lim$ sup $\mu$ iff some universal point subnet of $\mu$ converges to $x$; (v) defining $\mu \times \nu$ for any nets $\mu$ and $\nu$ to be $(\mu \times \nu)$ $\cdot(d, e)=\mu(d) \times \nu(e)$, we have $\lim \sup (\mu \times \nu)=(\lim \sup \mu) \times(\lim \sup \nu)$.

Presented to the Society November 18, 1955 under the title On Moore-Smith limits and filtered functions; received by the editors July 22, 1968. 
Approximate subnets. When $\mu$ and $\nu$ are nets in a uniform space we define $\nu$ to be an approximate subnet of $\mu$ if given any $F$ in $\mathcal{F}(\mu)$ and any member $\alpha$ of the uniformity, $\left[N_{\alpha}(F)\right]^{-}$is in $\mathcal{F}(\nu)$. (This would be the case if, for example, $\left(^{*}\right)$ given any final segment $I$ of the domain of $\mu$ and any $\alpha$ there is some final segment $J$ of the domain of $\nu$ such that for every $e$ in $J$ there is some $d$ in $I$ such that $\nu(e)$ $\subset N_{\alpha}(\mu(d))$.) If this is true, clearly $\lim \sup \nu \subset\left[N_{\alpha}(F)\right]-$ for every such $F$ and $\alpha$, and hence $\lim \sup \nu \subset \cap \bigcap_{F \alpha}\left[N_{\alpha}(F)\right]^{-}=\bigcap F=\lim \sup \mu$. This gives us the following basis for Duhamel theorems.

THEOREM D. If $\nu$ is an approximate subnet of $\mu$ then $\lim \sup \nu \subset$ $\lim \sup \mu$.

This yields, for example, the Duhamel theorem in [5] with weaker hypotheses; it also is the base for the equivalence of the Riemann and Darboux definitions of integrals for bounded functions on linear intervals (even to locally convex spaces). To see the latter we let $D$ be the set of all finite partitions of the interval and let $D$ ordered by refinement be $D_{\mu}$ and ordered by the partition norm be $D_{\nu}$. Let $\mu$ on $D_{\mu}$ and $\nu$ on $D_{\nu}$ assign to each $d$ the set of all Riemann sums of the function for the partition $d$. Obviously $\mu$ is a subnet of $\nu$, so $\lim \sup \mu$ $C \lim \sup \nu$. On the other hand, $\nu$ is an approximate subnet of $\mu$. This follows from the usual lemma-given any $d$ in $D_{\mu}$ and any $\epsilon>0$ there is some $\delta>0$ such that if $e$ is in $D_{\nu}$ and norm $e<\delta$ then $\nu(e) \subset N_{\epsilon}(\mu(d \bigvee e))$ where $d \bigvee e$ is the coarsest partition finer than both $d$ and $e$-since this simply asserts that $\left(^{*}\right)$ above is true. Thus $\lim \sup \mu=\lim \sup \nu$, which says more than is usually said about these integrals.

Total nets. We shall call a net total if each of its subnets has a cluster point. Clearly a net is total if it is finally compact or is convergent, and any subnet of a total net is total.

When $\mu$ is any function on a directed set to the space of subnets of a set $X$, the support of $\mu$ is $\{d: \mu(d) \neq \varnothing\}$; if the support of $\mu$ is a final segment of the domain of $\mu, \mu$ is finally a net; if the support is cofinal, $\mu$ is cofinally a net. If the kernel of $\mu$ refers to $\mu$ restricted to its support, the kernel is a net when $\mu$ is cofinally a net, in which case we define $\lim \sup \mu$ to be $\lim$ sup of the kernel of $\mu$, and $\mu$ to be total if the kernel of $\mu$ is total.

Theorem A. If $\mu$ is a total net in $X$ and $f$ is continuous on $X$ to $Z$, then $f(\mu)$ is total in $Z$; if $Z$ is Hausdorff, $f(\lim \sup \mu)=\lim \sup f(\mu)$.

Let $\nu$ be any subnet of $f(\mu)$. On the product of the domains of $\mu$ and $\nu$ define $\sigma^{\prime}$ by $\sigma^{\prime}(d, e)=\mu(d) \cap f^{\leftarrow}(\nu(e))$. Since $\nu$ is a subnet of $f(\mu)$, 
$\sigma^{\prime}$ is cofinally a net; and if $\sigma$ is the kernel of $\sigma^{\prime}$ then $\sigma$ is a subnet of $\mu$ and $f(\sigma)$ a subnet of $\nu$. Since $\mu$ is total, $\sigma$ clusters at some point $x^{\prime}$ and hence $f(\sigma)$ clusters at $f\left(x^{\prime}\right)$, so that $f\left(x^{\prime}\right) \in \lim \sup f(\sigma) \subset \lim \sup \nu$. Thus $f(\mu)$ is total. If $y \in \lim \sup f(\mu)$ we can choose a subnet $\nu$ of $f(\mu)$ that converges to $y$, and then construct $\sigma$ as above; since $f(\sigma)$ is a subnet of $\nu, f(\sigma)$ converges to $y$. But $f(\sigma)$ clusters at $f\left(x^{\prime}\right)$, so that if $Z$ is Hausdorff we must have $y=f\left(x^{\prime}\right)$ and so $y \in f(\lim$ sup $\mu)$ since $x^{\prime} \in \lim \sup \sigma \subset \lim \sup \mu$. Thus $f(\lim \sup \mu) \supset \lim \sup f(\mu)$. The reverse inclusion comes from the continuity of $f$.

We can state a more general version of Theorem A. Note that here $f(\mu)$ is not necessarily a net but is finally a net.

Theorem B. If $\mu$ is a total net in $X$ and $G$ is open in $X$ and $\lim \sup \mu \subset G$, then $\mu$ is finally in $G$ : if $f$ is continuous on $G$ to $Z$, then $f(\mu)$ is total and if $Z$ is Hausdorff, $f(\lim \sup \mu)=\lim \sup f(\mu)$.

If $\mu$ is not finally in $G$ it repeatedly intersects $X \backslash G$; this defines in $X \backslash G$ a subnet $\nu$ of $\mu$. Since $\mu$ is total $\nu$ clusters at some $x^{\prime}$ and clearly $x^{\prime} \in X \backslash G$. But $x^{\prime} \in \sup \lim \nu \subset \lim \sup \mu \subset G$. Thus $\mu$ is finally in $G$. If we let $D=\{d: \mu(d) \subset G\}$ then $D$ is a final segment of the domain $D_{\mu}$ of $\mu$, and if we let $\mu_{G}$ be $\mu$ restricted to $D$ then $\mu_{G}$ is a total net in $G$ and hence $f\left(\mu_{G}\right)$ is total in $Z$ by Theorem A. But $f\left(\mu_{G}\right)$ coincides with the kernel of $f(\mu)$ on a final segment of $D_{\mu}$ and so that kernel is total and hence $f(\mu)$ is. If $Z$ is Hausdorff then, using Theorem A, $f(\lim \sup \mu)=f\left(\lim \sup \mu_{G}\right)=\lim \sup f\left(\mu_{G}\right)=\lim$ sup kernel $f(\mu)=$ $\lim \sup f(\mu)$.

The conclusions of Theorem B obviously hold for any finally compact net and hence for any net in any compact $X$, provided $G$ is open and $\lim$ sup $\mu \subset G$.

Turning to product spaces, if $\mu$ is a net in $X$ and $\nu$ a net in $Y$, we have recalled that $(\lim \sup \mu) \times(\lim \sup \nu)=\lim \sup (\mu \times \nu)$. If $\mu \times \nu$ is total in $X \times Y$ and $p_{1}, p_{2}$ are the projections onto $X$ and $Y$ then each $p_{i}(\mu \times \nu)$ is total by Theorem A. Since $\mu$ is a subnet of $p_{1}(\mu \times \nu)$ and $\nu$ of $p_{2}(\mu \times \nu)$, both $\mu$ and $\nu$ are total. Conversely, since clearly a net is total iff each of its universal point subnets converges, the standard simple proof of the Tychonoff theorem, involving universal nets, shows that $\mu \times \nu$ is total if $\mu$ and $\nu$ are. From these remarks and Theorem $\mathrm{B}$ we have this consequence.

Theorem C. If $\mu$ is total in $X$ and $\nu$ total in $Y$ and $f$ is continuous on an open subset $G$ of $X \times Y$ to $Z$ and $(\lim \sup \mu) \times(\lim \sup \nu) \subset G$, then $\mu \times \nu$ is total and is finally in $G, f(\mu \times \nu)$ is total, and if $Z$ is Hausdorff, $f((\lim \sup \mu) \times(\lim \sup \nu))=\lim \sup f(\mu \times \nu)$. 
As an obvious special case there is

Corollary 1. The conclusions of Theorem $\mathrm{C}$ hold if $X$ and $Y$ are compact, $f$ is continuous on open $G$ in $X \times Y$ to $Z$, and $\mu$ and $\nu$ are nets in $X$ and $Y$ such that $(\lim \sup \mu) \times(\lim \sup \nu) \subset G$.

For a real variables application of Corollary 1 , let $\bar{R}$ be the extended reals, let $G$ be $\bar{R} \times \bar{R}$ with $(-\infty, \infty)$ and $(\infty,-\infty)$ deleted, and let $f$ on $G$ be addition. For any two sets $A$ and $B$ in $\bar{R}$ let $A+B$ $=f((A \times B) \cap G)$. For any two nets $\mu, \nu$ in $\bar{R}$ define $\mu \oplus \nu$ on $D_{\mu} \times D_{\nu}$ by $(\mu \oplus \nu)(d, e)=\mu(d)+\nu(e)$, and if $D_{\mu}=D_{\nu}$ let $\mu+\nu$ on $D_{\mu}$ be $(\mu+\nu)(d)$ $=\mu(d)+\nu(d)$. Both functions may fail to be cofinally nets; but if $\mu+\nu$ is cofinally a net then so is $\mu \oplus \nu$, the kernel of $\mu+\nu$ is a subnet of the kernel of $\mu \oplus \nu$, and

$$
\text { lim sup (kernel }(\mu+\nu)) \subset \lim \sup (\operatorname{kernel}(\mu \oplus \nu)) \text {. }
$$

Thus, if for any function $\rho$ on a directed set to the subsets of $R$ that is cofinally a net we denote the greatest element of lim sup (kernel $\rho$ ) by $\sup \lim \rho$ and the least by $\inf \lim \rho$, we have

$$
\sup \lim (\mu \oplus \nu) \geqq \sup \lim (\mu+\nu) \geqq \inf \lim (\mu+\nu) \geqq \inf \lim (\mu \oplus \nu)
$$

if $\mu+\nu$ is cofinally a net. We recall that if $\mu$ and $\nu$ are nets in $\bar{R}$ and sup $\lim \mu+\sup \lim \nu$ is defined it must be the greatest element of $\lim \sup \mu+\lim \sup \nu$, and dually for inf $\lim$. The following now easily results from Corollary 1.

Corollary 2. If $\mu$ and $\nu$ are nets in $\bar{R}(\lim \sup \mu) \times(\lim \sup \nu)$ contains neither $(-\infty, \infty)$ nor $(\infty,-\infty)$, then (i) $\mu \times \nu$ is finally in $G$ and $\mu \oplus \nu$ is finally $a$ net, (ii) $\lim \sup \mu+\lim \sup \nu=\lim \sup (\mu \oplus \nu)$ and hence sup $\lim \mu+\sup \lim \nu=\sup \lim (\mu \oplus \nu) \geqq \inf \lim (\mu \oplus \nu)=$ inf $\lim \mu+\inf \lim \nu$.

Clearly the first part of (ii) is the heart of theorems connecting addition with sup lim and inf lim of nets in real variables: partial sums, difference quotients, approximation sums for integrals, etc. Results similar to the above can be obtained by replacing addition by multiplication or by any other continuous binary operation defined on an open subset of $\bar{R} \times \bar{R}$.

The above results can be applied in a variety of situations; for example, if $X$ and $Y$ are locally convex Hausdorff spaces and $\mu$ and $\nu$ are total in $X$ and $Y$ then $(\lim \sup \mu) \otimes(\lim \sup \nu)=\lim \sup (\mu \otimes \nu)$, $\otimes$ denoting tensor product. If $X$ is a real linear topological space with sufficiently many continuous linear functionals and $\mu$ is a net 
in $X$ that finally lies in some compact convex set then conv $(\lim \sup \mu)$ $=$ conv $(\lim \sup (\operatorname{conv} \mu))$ where "conv" denotes the closed convex cover [2, p. 133]. For both sets are closed convex compact, and by the separation theorem it is enough to show that $f(\operatorname{conv}(\lim \sup \mu))$ $=f(\operatorname{conv}(\lim \sup (\operatorname{conv} \mu)))$ for every $f$ in the dual $X^{*}$; since both sets are compact and $f$ is continuous linear, $f$ and conv commute so it must be shown that conv $f(\lim \sup \mu)=\operatorname{conv} f(\lim \sup (\operatorname{conv} \mu))$. But from Theorem A this is equivalent to conv $\lim \sup f(\mu)=$ conv $\lim \sup f(\operatorname{conv} \mu)=$ conv $\lim \sup \operatorname{conv} f(\mu)$, and this is the easily verified special case in which $X=R$. Thus if $\nu$ is a finally compact net of closed convex sets in a real linear topological space whose adjoint separates points and if $\mu(d)=$ extreme points of $\nu(d)$ for every $d$ in $D_{\nu}$, then conv $\lim \sup \mu=\mathrm{conv} \lim \sup \nu$, and if $X$ is locally convex Hausdorff then also every extreme point of conv $\lim \sup \nu$ is in $\lim \sup \mu$. These are well known and due to Jerison [1].

As a final application let $\mu$ and $\nu$ be nets in a topological space $X$ possessing a continuous binary operation o. If $\mathcal{F}(\mu) \subset \mathcal{F}(\mu \circ \nu)$ then $\lim \sup \mu \supset \lim \sup (\mu \circ \nu) \supset(\lim \sup \mu) \circ(\lim \sup \nu)$, the last inclusion from the continuity of the operation. Thus if $\mathcal{F}(\mu) \subset \mathcal{F}(\mu \circ \mu)$ then $\lim \sup \mu$ is a subsemigroupoid (subsemigroup if $\circ$ is associative) and, if $\mathfrak{F}(\nu) \subset \mathfrak{F}(\mu) \subset \mathcal{F}(\mu \circ \nu)$, then $\lim \sup \mu$ is a right ideal of $\lim \sup \nu$.

If $\circ$ is associative, $X$ Hausdorff, $\mu$ total in $X$, and $\lim$ sup $\mu$ compact and a subsemigroup, then $\lim \sup \mu$ is a compact subgroup if either of these is true: (i) $\mathcal{F}(\mu)$ contains $\mathcal{F}(\mu \circ \nu)$ and $\mathcal{F}(\nu \circ \mu)$ for every universal point subnet $\nu$ of $\mu$; (ii) $\mathcal{F}(\mu)$ contains $\mathcal{F}(x \circ \mu)$ and $\mathcal{F}(\mu \circ x)$ for every $x$ in a dense subset of lim sup $\mu$. For in either case we want $x \circ \lim \sup \mu \supset \lim \sup \mu$ and $(\lim \sup \mu) \circ x \supset \lim \sup \mu$, for every $x$ in $\lim \sup \mu$. If (i) holds we choose a universal point subnet $\nu$ of $\mu$ that converges to $x$; then $x \circ \lim \sup \mu=(\lim \sup \nu) \circ(\lim \sup \mu)=$ $\lim \sup (\nu \circ \mu) \supset \lim \sup \mu$ by Corollary 1 and (i); the dual follows similarly. If (ii) holds we have $x \circ \lim \sup \mu=\lim \sup (x \circ \mu) \supset \lim \sup \mu$, and dually, by Theorem A and (ii), for every $x$ in a dense subset of $\lim \sup \mu$; since $\lim \sup \mu$ is compact and $X$ is Hausdorff these must hold for every $x$ in $\lim \sup \mu$.

The latter of the above two propositions immediately leads to this: if $\circ$ is associative and continuous and $X$ Hausdorff, if $\mu$ is monotone decreasing (for example, the identity net on a filter base) and finally compact, then $\lim \sup \mu$ is a compact subgroup if, given any $d$ in the domain $D$ of $\mu$ and any $x$ in $\mu(d)$, there are $d^{\prime}, d^{\prime \prime}$ in $D$ such that $[\mu(d)]^{-} \supset \mu\left(d^{\prime}\right) \circ \mu\left(d^{\prime}\right)$ and $\left(x \circ[\mu(d)]^{-}\right) \cap\left([\mu(d)]^{-} \circ x\right) \supset \mu\left(d^{\prime \prime}\right)$. This is essentially the basic theorem for compact semigroups due to Peck [3], [6]. 


\section{REFERENCES}

1. M. Jerison, $A$ property of extreme points of compact convex sets, Proc. Amer. Math. Soc. 5 (1954), 782-783.

2. J. L. Kelley, I. Namioka et al., Linear topological spaces, Van Nostrand, Princeton, N. J., 1963.

3. J. E. L. Peck, An ergodic theorem for a noncommutative semigroup of linear operators, Proc. Amer. Math. Soc. 2 (1951), 414-421.

4. B. J. Pettis, On Moore-Smith limits and filtered functions, Abstract, Bull. Amer. Math. Soc. 62 (1956), 30.

5. A. E. Taylor, Advanced calculus, Ginn, Boston, Mass., 1955.

6. A. D. Wallace, The structure of topological semigroups, Bull. Amer. Math. Soc. 61 (1955), 95-112.

University of North Carolina, Chapel Hill 